\title{
Investigating the Factors Affecting the Development of Biliary Pancreatitis and Their Relationship with the Type and Severity of Complications
}

\author{
Amirhossein Pirouz ${ }^{1}$, Ehsan Sadeghian ${ }^{1, *}$, Mehdi Jafari ${ }^{1}$, Reza Eslamian ${ }^{1}$, Fezzeh Elyasinia ${ }^{1}$, \\ Mohammad Ali Mohammadi-Vajari ${ }^{1}$, Ali Ghorbani Abdehgah ${ }^{1}$, Ahmadreza Soroush ${ }^{1}$
}

1. Department of Surgery, Shariati Hospital, Tehran University of Medical Sciences

\section{* Corresponding Author:}

Ehsan Sadeghian ,MD

Shariati Hospital, Jalal-e-Al-e-Ahmad Hwy, North Kargar St., Tehran, Iran

Tel: + 982184902455

Fax: + 982188633039

Email: ehsan810@yahoo.com

Received: 18 Jun. 2020

Accepted: 09 Nov. 2020

\section{ABSTRACT}

\section{BACKGROUND}

Pancreatitis is an inflammation of the pancreatic tissue. Gallstones are known to be the most common cause of acute pancreatitis, especially in eastern countries, including Iran. Pancreatitis, in its course, can cause complications for the patient. Different systems have been identified as predictors of the severity of acute pancreatitis. As a result, we decided to examine the factors influencing the severity of biliary pancreatitis and their relationship with the complications in Iranian society.

\section{METHODS}

The present study is a cross-sectional, analytical study that was performed retrospectively on 160 patients with biliary pancreatitis. The main and dependent variable in this study is the severity of pancreatitis, which is divided into two groups of complications (local complications and systemic complications) and without complications. The necessary information was extracted from the patients' files and evaluated with SPSS software version 22.

\section{RESULTS}

Based on the results of single-variable analysis, there was a significant relationship between the patient's age, sex, Ranson and CRP criteria, and complication of the disease. In the univariate analysis, no significant statistical relationship was found between patients' BMI(Body Mass Index), CBD (common bile duct) size, serum alkaline phosphatase level, gallstone size, and FBS(Fasting blood sugar), and the complications of the disease, based on the multivariate analysis results.

\section{CONCLUSION}

The results of this study showed that four variables of the female sex, stone size, $\mathrm{CRP}$, and high score of Ranson criteria act as independent risk factors in the development of complicating biliary pancreatitis.

\section{KEYWORDS:}

Reflux, Manometry, Diaphragmatic breathing, Aerobic exercise, Quality of life, LES

\section{Please cite this paper as:}

Pirouz AR, Ghorbani Abdehgah A, Sadeghian E, Jafari M, Eslamian R, Eliasinia F, Mohammadi-Vajari MA, Soroush AR. Investigating the Factors Affecting the Development of Biliary Pancreatitis and Their Relationship with the Type and Severity of Complications. Middle East J Dig Dis 2021;13:43-48. doi: 10.34172/mejdd.2021.202. 


\section{INTRODUCTION}

Pancreatitis is an inflammation of the pancreatic tissue, which can be acute or chronic. Extensive epidemiological studies have been performed on the causes of acute pancreatitis and the severity of pancreatitis. ${ }^{1,2}$ Gallstones, chronic alcoholism, biliary tract disease, tumors, trauma, abdominal surgery, certain medications (such as chlorothiazide or drugs), corticosteroids, some infections such as mumps, hyperlipidemia, hyperparathyroidism, vasculitis, hypercalcemia, ERCP (endoscopic retrograde cholangiopancreatography), chronic renal failure, and dialysis are some of the causes of pancreatitis. ${ }^{3}$

Gallstones are known to be the most common cause of acute pancreatitis, especially in eastern countries, including Iran. Various systems, including APACHE-2, Ranson, Glasgow, and BISAP(Bedside Index for Severity in Acute Pancreatitis), have been identified as predictors of the severity of acute pancreatitis, and their efficacy has been studied. In a 2013 study, Khanna and colleagues examined and compared the criteria of MOSS(multiple organ system score), Glasgow, Ranson, SIRS(Systemic inflammatory response syndrome), BISAP, CTSI(modified CT severity index), APACHE-II (Acute Physiology And Chronic Health Evaluation II), CRP, IL-6, and procalcitonin in predicting the severity of pancreatitis, organ failure, and mortality. ${ }^{4,5}$ IL- 6 and CRP have been shown to play an important role in the early detection of pancreatitis and pancreatic necrosis, while APACHE-II and Ranson's criteria are effective in predicting pancreatic mortality. ${ }^{4,5}$ Kuo and co-workers in a review study looked at different systems for evaluating the severity of pancreatitis and showed that systems such as BISAP and HAPS(Harmless Acute Pancreatitis Score) and singleminer criteria could be useful in predicting the severity of pancreatitis in the first 24 hours. $^{5}$

Pancreatitis, in its course, can cause complications for the patient, the knowledge of which can play a significant role in the treatment of patients. Side effects of pancreatitis include pancreatic necrosis, pseudocyst, pancreatic abscess, a secondary bacterial infection of the pancreas, pulmonary and diabetes complications, and chronic calcium deficiency. Despite extensive studies on the severity of pancreatitis and its causes, ${ }^{6}$ studies on the relationship between complications and factors determining the severity of pancreatitis, especially in Iranian society, are very few. ${ }^{7,8}$
As a result, we decided to investigate the factors affecting the severity of biliary pancreatitis and their relationship with the complications of this disease.

\section{MATERIALS AND METHODS}

The present study is a cross-sectional, analytical retrospective study of 160 patients with biliary pancreatitis. All adult patients who were referred to Shariati Hospital due to pancreatitis in the period of 2017-2020 were studied. The population of patients included patients with acute biliary pancreatitis who sought or referred to the emergency department of Shariati Hospital following the onset of symptoms, then were admitted to the hospital and were diagnosed with acute biliary pancreatitis during examinations, and medical and supportive measures were started for them.

Studied variables included: patient age, sex, BMI, imaging findings (ultrasound, EUS (Endoscopic ultrasound), MRCP (Magnetic resonance cholangiopancreatography), computerized tomography (CT)scan, ERCP), Alkaline Ph, WBC (white blood cell), FBS (fasting blood sugar), CRP (C-reactive protein), serum amylase, CBD (common bile duct) size, stone size, and Ranson criteria. The main and dependent variable in this study was the severity of pancreatitis, which was divided into two groups with and without complications. Complications studied in the present study included: local complications (includingpancreatic necrosis, pseudocyst, pancreatic ascites, abscess, and pancreatic phlegmon), systemic complications (including: pulmonary, cardiac, neurological, metabolic, gastrointestinal, and renal complications), bleeding, pleural effusion, pneumonia, pericardial effusion, hyperglycemia (FBS above $200 \mathrm{mg} / \mathrm{dL}$ (milligrams per decilitre)or FBS above 125 in patients who have not previously had diabetes), hypocalcemia, pulmonary embolism, subcutaneous tissue necrosis, coagulopathy, and mortality.

Sampling was performed by easy (census) method, so that all patients registered in the study period were included in the study, provided that they had entry criteria. The necessary information, including independent and dependent variables, was extracted from the archived files of the patients.

\section{Data analysis}

The relationship between the factors affecting biliary 
pancreatitis and whether or not pancreatitis was complicated was evaluated using $t$ test, Chi-square, Mann Whitney U test, Fisher exact test, and cross-sectional table methods. Then, statistical methods such as regression modeling and classification were used to eliminate distorting factors or other effective factors that may affect the results. SPSS software version 22 was used to analyze the data. Also, the first error level of less than $5 \%$ was considered as a significant level.

\section{RESULTS}

A total of 160 patients with a mean age of $54.17 \pm 17.76$ years were examined in this study, which included 57 (35.6\%) men and 103 (64.4\%) women (77 patients with a complication of biliary pancreatitis), and 83 patients with uncomplicated biliary pancreatitis). Over 3.5 years, there were 160 cases of pancreatitis, 50 of which had a history of gallstones but had not been operated on. In this center, 62 of them were subjected to cholecystectomy in the same hospital.

The most common complications in the patients examined were pancreatic necrosis (20 cases), pseudocyst (20 cases), and ascites (15 cases), respectively. Other complications included hyperglycemia (7 cases), renal failure (12 cases), pulmonary embolism (5 cases), gastrointestinal (GI) bleeding (3 cases), severe coagulopathy (5 cases), abscess and phlegmon (10 cases), and respiratory system complications with 30 cases (pneumonia, atelectasis, ARDS(Acute respiratory distress syndrome), and pleural effusion). Also, the most common symptoms of patients at the time of referral were pain, nausea, and vomiting.

According to the findings of this study, there was a strong and significant relationship between age $(p<0.001)$ and sex $(p=0.034)$ of the patients and complication of the disease, so that with age, patients were more likely to have complications. Increasingly, the highest incidence occurred in patients over 60 years of age and in women. Also, the analysis of the obtained data showed that there was a strong and significant statistical relationship between the mean Ranson criterion $(p<0.001)$ at the beginning of the study and the CRP level ( $p=0.024)$ of the patients with the complication of the disease. Elevated Ranson criteria in patients also increase the risk of complications. In this study, the mean stone size in the complication group was $4.51 \mathrm{~mm}$ and in the uncomplicated group was $3.63 \mathrm{~mm}$, so that with increasing stone size and the number of stones
Table 1: The relationship between various factors and the occurrence of complication-free and uncomplicated pancreatitis

\begin{tabular}{|c|c|c|c|c|}
\hline \multirow{2}{*}{ Variables } & \multirow{2}{*}{ Level } & Non-complicated & Complicated & \multirow{2}{*}{$p$ value } \\
\hline & & $\mathrm{N}=82$ & $N=77$ & \\
\hline \multirow{4}{*}{$\begin{array}{l}\text { Age } \\
\text { groups }\end{array}$} & $20-40$ & $11(24)$ & $35(76)$ & \multirow{4}{*}{$<0.001$} \\
\hline & $40-60$ & $16(40)$ & $24(60)$ & \\
\hline & $60-80$ & $45(65)$ & $24(35)$ & \\
\hline & $80-100$ & $5(100)$ & 0 & \\
\hline \multirow{2}{*}{ Sex } & Male & $21(37)$ & $36(63)$ & \multirow{2}{*}{0.034} \\
\hline & Female & $56(55)$ & $47(45)$ & \\
\hline \multirow{2}{*}{ BMI } & Mean & 23.32 & 22.36 & \multirow{2}{*}{0.090} \\
\hline & SD. & 3.73 & 3.39 & \\
\hline \multirow{2}{*}{ CBD } & Mean & 14.52 & 9.62 & \multirow{2}{*}{0.072} \\
\hline & SD. & 2.17 & 1.05 & \\
\hline \multirow{2}{*}{$\begin{array}{l}\text { Ranson's } \\
\text { criteria }\end{array}$} & Mean & 4.6 & 3.4 & \multirow{2}{*}{$<0.001$} \\
\hline & SD. & 1.1 & 1.1 & \\
\hline \multirow{2}{*}{$\mathrm{ALP}^{\dagger}$} & Mean & 479.2 & 388.9 & \multirow{2}{*}{0.069} \\
\hline & SD. & 100 & 80.9 & \\
\hline \multirow{2}{*}{$\begin{array}{l}\text { Size of } \\
\text { stone }\end{array}$} & Mean & 4.5 & 3.6 & \multirow{2}{*}{0.090} \\
\hline & SD. & 2 & 1.9 & \\
\hline \multirow{2}{*}{ FBS } & Mean & 166 & 97.8 & \multirow{2}{*}{0.082} \\
\hline & SD. & 27.4 & 15.7 & \\
\hline \multirow{2}{*}{ CRP } & Mean & 46.1 & 40.5 & \multirow{2}{*}{0.024} \\
\hline & SD. & 6.4 & 6.7 & \\
\hline
\end{tabular}

${ }^{\dagger}$ Alkaline phosphatase, BMI(Body Mass Index), CBD (common bile duct), FBS(fasting blood sugar), CRP(C-reactive protein)

(more than two cases), the probability of complication also increased $(p=0.009)$. There was no significant statistical relationship between mean blood sugar (FBS) and BMI of the studied patients and complications of the disease (mean FBS in the complication group was 166 and in the uncomplicated group was 97.84) $(p=0.081$ and $p=0.090)$ (Table 1).

Also, the results of multivariate regression modeling showed that among the studied variables, four main variables such as female sex, Stone size, CRP, and Ranson criterion act as independent risk factors in the development of complicating biliary pancreatitis. Among the factors studied, the Ranson criterion was the strongest factor, and the size of the stone was the weakest independent risk factor.

\section{DISCUSSION}

In this study, 160 people were studied; according to the history of patients, abdominal pain was the most common among the symptoms complained of by the patients. Our 
findings showed a strong and significant relationship that was observed between the age of the patients and female sex with the complications of the disease $(p<0.001$ and $p=0.034$, respectively). This finding was consistent with the findings of other researchers. For example, a study conducted in the United States by Dhiraj Yadav and colleagues on the epidemiology of pancreatitis and its risk factors showed that the disease severely affected patients' quality of life and the overall incidence of pancreatitis in blacks and women was relatively high.,

In the study of Baghi and co-workers in Rasht, the ratio of women to men was 1.599 , and the highest age prevalence in the age group was 41-50 years. However, no significant relationship was observed between the patients' BMI and pancreatitis complications in the present study $(p=0.90){ }^{8}$ In a 2012 study in Sweden conducted by $\mathrm{O}$ Sadr-Azodi on 68,000 people over a period of about 12 years, during which 424 people developed acute pancreatitis, the study's findings showed that waist size more than $105 \mathrm{~cm}$ compared with people with waist circumference 75.1 to $85 \mathrm{~cm}$ doubled the risk. This association was seen in both pancreatitis caused by gallstones and unrelated to gallstones, but there was no association between high BMI and the risk of developing acute pancreatitis, which was consistent with the results of our study. But a study by Leonilde Bonfrate in Italy examined the prognosis of gallstones and pancreatitis. It has been shown that rapid weight loss, whether through a low-calorie diet or bariatric surgery, is a risk factor for this complication. Prophylactic treatment during weight loss in obese people is recommended with ursodeoxycholic acid. ${ }^{9}$

Based on the results of this study, no statistically significant relationship was observed between CBD size and disease complication ( $p=0.072$ ), the mean stone size in the complication group was 4.51 and in the uncomplicated group was $3.63 \mathrm{~mm}(p=0.072)$, so that $30 \%$ of patients had more than two stones (multiple stones), 10\% had two stones, $40 \%$ had one stone $(25 \%<1 \mathrm{~cm}, 10 \%, 1-2$, and $5 \%>2 \mathrm{~cm}$ ) and $20 \%$ had slag. This finding was consistent with the results of other studies, but some studies have yielded different results. For example, Dhiraj Yadav and others in a study conducted in the United States showed that gallstones were the most common cause of pancreatitis and related complications. So, cystectomy reduced the risk of future attacks.
This study also showed that alcohol was the most common cause of chronic pancreatitis, and smoking as a non-dependent factor in the development of acute and chronic pancreatitis. ${ }^{2}$ Also, based on the results of a singlevariable analysis in the present study, it was shown that there is a significant difference between the mean score of Ranson criterion in the two groups (4.60 in the complication group and 3.41 in the uncomplicated group), but between the amount of alkaline phosphatase, FBS, WBC, serum amylase, and disease complications did not show a strong and significant association ( $p>0.05)$. In a 2003 study by Alimoglu $\mathrm{O}$ on 43 patients with acute biliary pancreatitis in Turkey, which was done retrospectively to evaluate the results of cystectomy at the time of hospitalization in patients with acute biliary pancreatitis, the patients were divided into two general categories. The first group consisted of 27 patients who underwent acute cholecystectomy after the first admission of acute biliary pancreatitis, and the second group consisted of 16 patients who underwent cystectomy after the recurrence of biliary pancreatitis. 24 patients in the first group had less than three Ranson's criteria, while 12 patients in the second group had this condition. Another study of 76 patients with biliary pancreatitis at a referral center in the United States looked at the risk factors for severe complications of biliary pancreatitis. 55\% had multiple gallstones, $21 \%$ had biliary slag, and 24\% had structural problems.

The findings showed that the mean amylase and alkaline phosphatase levels were $64 \%$ and $49 \%$ higher in biliary pancreatitis, respectively than in other etiologies. The study showed that necrosis of the pancreas, ascites, respiratory, and renal complications were most prevalent, which was somewhat consistent with our study so that in our study, the most common complications in the examined patients included necrosis of the pancreas (20 cases). There were pseudocysts (20 cases) and ascites (15 cases). Other complications include hyperglycemia (7 cases), renal failure (12 cases), pulmonary embolism ( 5 cases), GI bleeding ( 3 cases), severe coagulopathy ( 5 cases), abscess and phlegmon (10 cases), and respiratory system complications with 30 cases (pneumonia, atelectasis, ARDS, pleural effusion). In some studies, alkaline phosphatase has been shown to be an unrelated predictor of long-term complications of biliary pancreatitis $(\mathrm{OR}=6.69, p<0.001)$, which is inconsistent with our study. Based on the results 
of multivariate regression, in our study, four variables of the female sex, stone size, CRP, and Ranson's criteria were identified as independent risk factors for complications of biliary pancreatitis. However, other factors, such as the age of the patients, although in a single-variable analysis, had a significant relationship with the complication of pancreatitis, but their role as an independent risk factor was not proven based on the information obtained.

According to various studies, metabolic disorders and unknown factors play an important role in the etiology of biliary pancreatitis complications. Paying attention to these risk factors can accelerate the healing and recovery of patients. For example, the results of our study showed that between average blood sugar (FBS), there is a relationship between the patients studied and the complication of the disease (the mean FBS was $166 \mathrm{mg} / \mathrm{dL}$ in the complication group and $97.84 \mathrm{mg} / \mathrm{dL}$ in the uncomplicated group), although it was not statistically significant $(p=0.081)$. In a 2005 study in Gilan by Iraj Baghi and his colleague to examine the causes of acute pancreatitis in patients admitted to the hospital, $49 \%$ of the patients did not have a specific cause for pancreatitis (idiopathic). ${ }^{8}$

Insufficient attention to previous drug use, including the use of NSAIDs and other drugs, and the lack of attention to other common causes of pancreatitis, including metabolic causes, appear to justify this difference. The most commonly known cause of acute pancreatitis was gallstones, which are different from those in the United States and many European countries. ${ }^{9}$

In our study, the mean CRP in the complication group was $46.16 \mathrm{mg} / \mathrm{L}$ and in the uncomplicated group was 44.56 $\mathrm{mg} / \mathrm{L}$. The results of the single-variable analysis showed that increasing the CRP level in patients had a significant effect on the probability of complicating the disease ( $p=$ 0.024), which is consistent with the findings of other researchers. ${ }^{10,11}$ However, some studies have not shown a relationship, for example, in the study of Fateh and coworkers, which was conducted to investigate the relationship between serum CRP and interleukin 15 and 17 levels with severe biliary pancreatitis, the results showed that serum levels of interleukin 17 in determining the severity of acute pancreatitis was an important factor according to Ranson's criteria, but there was no association between serum levels of interleukin 15 and CRP in patients and acute pancreatitis and its possible side effects. ${ }^{12,13,14}$

\section{CONCLUSION}

The results of this study showed that the most common complications in the examined patients included pancreatic necrosis, pseudocyst, and ascites. Also, among the risk factors studied, four variables of the female sex, stone size, CRP, and Ranson criteria acted as independent risk factors in the development of complicating biliary pancreatitis. Among the factors studied, the Ranson criterion was the strongest factor, and the size of the stone was the weakest independent risk factor.

\section{ETHICAL APPROVAL}

There is nothing to be declared.

\section{CONFLICT OF INTEREST}

The authors declare no conflict of interest related to this work.

\section{REFERENCES}

1. Dervenis C. Assessments of severity and management of acute pancreatitis based on the Santorini Consensus Conference report. JOP 2000;1:178-82.

2. Portelli M, Jones CD. Severe acute pancreatitis: pathogenesis, diagnosis and surgical management. Hepatobiliary Pancreat Dis Int 2017;16;155-9. doi: 10.1016/ s1499-3872(16)60163-7.

3. Cappell MS. Acute pancreatitis: etiology, clinical presentation, diagnosis, and therapy. Med Clin North Am 2008;92:889-923. doi: 10.1016/j.mena.2008.04.013.

4. Khanna AK, Meher S, Prakash S, Tiwary SK, Singh U, Srivastava A, et al. Comparison of Ranson, Glasgow, MOSS, SIRS, BISAP, APACHE-II, CTSI Scores, IL6 , CRP, and procalcitonin in predicting severity, organ failure, pancreatic necrosis, and mortality in acute pancreatitis. HPB Surg 2013;2013:367581. doi: $10.1155 / 2013 / 367581$.

5. Asgari S, Mohaghegh P, Ghorbani-Abdehgah A, Molavi B, Yaghoobi-Notash A, Mir A, et al. Comparison of surgical site infections between laparoscopic and open cholecystectomy. Govaresh 2018;22:266-70.

6. Yadav D, Lowenfels AB. The epidemiology of pancreatitis and pancreatic cancer. Gastroenterology 2013;144:1252-61. doi: 10.1053/j.gastro.2013.01.068.

7. Amini M, Ahmadabadi A, Jand Y, Mosayebi G, Ghazavi A. Urine trypsinogen-2: A valuable factor for the diagnosis of acute pancreatitis. J Arak Uni Med Sci 2012;15:8-16. [in Persian]

8. Baghi I, Mohammadzadeh M. Relative Frequency of Causes of Acute Pancreatitis in Hospitalized Patients. Jour Guilan Uni Med Sci 2005;14:31-5. [in Persian] 
9. Roberts S, Akbari A, Thorne K, Atkinson M, Evans PA. The incidence of acute pancreatitis: impact of social deprivation, alcohol consumption, seasonal and demographic factors. Alim Pharmacol Ther 2013;38:539-48.

10. De Oliveira ML, Winter JM, Schafer M, Cunningham SC, Cameron JL, Yeo CJ, et al. Assessment of complications after pancreatic surgery: a novel grading system applied to 633 patients undergoing pancreaticoduodenectomy. Ann Surg 2006;244:931-7. doi: 10.1097/01. sla.0000246856.03918.9a.

11. Fateh S, Mamaghani MR, Mosayebi G. The relationship between serum levels of interleukin 15 and 17 and severity of acute pancreatitis. J Arak Uni Med Sci 2012;15:70-6. [in Persian]

12. Fisic E, Poropat G, Bilic-Zulle L, Licul V, Milic S, Stimac D. The role of IL-6, 8, and 10, sTNFr, CRP, and pancreatic elastase in the prediction of systemic complications in patients with acute pancreatitis. Gastroenterol Res Pract 2013;2013:282645. doi: 10.1155/2013/282645.

13. Cardoso FS, Ricardo LB, Oliveira AM, Canena JM, Horta DV, Papoila AL. C-reactive protein prognostic accuracy in acute pancreatitis: timing of measurement and cutoff points. Eur J Gastroenterol Hepatol 2013;25:784-9. doi: 10.1097/MEG.0b013e32835fd3f0.

14. Chen SM, Xiong GS, Wu SM. Is obesity an indicator of complications and mortality in acute pancreatitis? An updated meta-analysis. J Dig Dis 2012;13:244-51. doi: 10.1111/j.1751-2980.2012.00587.x. 